\title{
Ökonomisches Nirwana - Die Schuldenbremse führt zu weiterer gesellschaftlicher Spaltung
}

Heinz-J. Bontrup

Nach einer Übergangsphase, die 2011 beginnt, sollen ab 2020 die Haushalte der Bundesländer in „normalen“ Konjunkturzeiten keine Schulden mehr machen dürfen. Für die Übergangszeit von 2011 bis 2020 plant man, die Neuverschuldungen stufenweise zurückzuführen. Die armen Bundesländer ${ }^{1}$ erhalten zur Begleichung ihrer Altschulden neun Jahre lang jährlich 800 Mio. $€$ aus einem Topf von Bund und reichen Ländern. Dem Bund erlaubt man zukünftig noch eine maximale Neuverschuldung bis $0,35 \%$ bezogen auf das Bruttoinlandsprodukt pro Jahr. Dies sind nach heutigem Stand etwa rund 8,5 Mrd. €. Ein solch niedriger Wert wurde in den letzten 18 Jahren seit der Wiedervereinigung in keinem Jahr auch nur annähernd erreicht. Die jahresdurchschnittliche Verschuldung des Bundes lag zwischen 1991 und 2008 bei $29,8 \mathrm{Mrd}$. $€$ und die Verschuldung inklusive der Länder, Gemeinden und Sozialversicherung bei jahresdurchschnittlich 45,4 Mrd. €.

Die von der Föderalismuskommission II gemachten Vorgaben gelten dabei nicht für jedes einzelne Jahr, sondern für einen Konjunkturzyklus. Im Abschwung darf der Bund noch einen Kredit aufnehmen, der aber im nächsten Aufschwung sofort zurückzuzahlen ist. Ausnahmen sind nur noch für Naturkatastrophen und schwere Wirtschaftskrisen, wie beispielsweise in der jetzigen Weltwirtschaftskrise, vorgesehen. Das ganze Werk sei eine „Sternstunde des Föderalismus", so der SPD-Fraktionschef Peter Struck, und soll verfassungsmäßig im Grundgesetz festgeschrieben werden. Kommt es zu dieser „fundamentalen Weichenstellung" (Bundeskanzlerin Angela Merkel (CDU)), zu einer "Wetterwende“ (Horst Seehofer (CSU)), und das Ganze geht im Sommer durch den Bundestag und Bundesrat, dann findet quasi Wirtschaftspolitik in Deutschland, dem wirtschaftlich größten Land in der Europäischen Union (EU), nicht mehr statt.

Die Geldpolitik wurde mit dem Euro bereits nationalstaatlicher Kontrolle entzogen. Jetzt schaffen CDU/CSU und SPD mit Unterstützung der FDP und Bündnis 90/Die Grünen in Deutschland auch noch die den Euroländern verbliebende Fiskalpolitik ab. Denn mit der geplanten „Schuldenbremse" - sinnvoller wäre nach Peter Bofinger eine „Steuersenkungsbremse“ - ist in Zukunft eine seriöse und rationale antizyklische Fiskalpolitik nicht mehr möglich. Bei 0,35\% Verschuldungsspielraum reicht es nicht einmal mehr aus, die automatischen Konjunkturstabilisatoren voll wirken zu lassen. Hier verzichtet bekanntlich der Staat bei einem Wachstumseinbruch darauf, die wegbrechenden Steuereinnahmen durch entsprechende Staatsausgabensenkungen zu kompensieren. Kein anderes EU-Land verhält sich wirtschaftspolitisch mit einer "Schuldenbremse" so borniert wie die Deutschen. Selbst die neoliberal ausgerichtete EU erlaubt im „Europäischen Stabilitäts- und Wachstumspakt" eine Neuverschuldung in Höhe von 3 \% des Bruttoinlandsprodukts. Und schon diese „Schuldenbremse" ist zu Recht von dem ehemaligen Kommissionspräsidenten und Wirtschaftswissenschaftler Romano Prodi als „dumm und töricht" bezeichnet worden. Der Staat ist nun einmal „Gefangener" und gleichzeitig „Akteur“ auf dem „kapitalistischen Spielfeld“. Er wird unweigerlich mit in den Sog einer jeden, immanent den Marktgesetzen unterliegenden Konjunktur gezogen. Will sich der Staat hier nicht prozyklisch, also krisenverschärfend verhalten, so bleibt ihm im Abschwung nur, die konjunkturbedingte Staatsverschuldung hinzunehmen, die sich im Aufschwung wieder abbaut.

Strukturelle - überzyklische - Staatsverschuldung ist hiervon unabhängig $\mathrm{zu}$ sehen. Diese kann nur durch eine adäquate Steuererhebung und durch staatliche Abgaben bekämpft werden. Hier haben in Deutschland neoliberale Politiker den Staat durch Privatisierungen und völlig überzogene Steuersenkungen für Unternehmer, Besserverdienende und Vermögende bei gleichzeitigen Sozialkürzungen für die Schwächsten in unserer Gesellschaft vor die Wand gefahren. Die Senkung der Staatsquote wurde zum deutschen Mantra. Der größte Teil der insgesamt bei den Gebietskörperschaften (Bund, Länder und Gemeinden) aufgelaufenen Staatsverschuldung in Höhe von rund 1,5 Bill. €, dies sind gut $65 \%$ des Bruttoinlandsprodukts, ist dabei nicht nur einer widersprüchlichen kapitalistischen Ökonomie, sondern auch einer falschen Politik geschuldet. Außerdem muss man bei der aufgelaufenen Staatsverschuldung den Sonderfall der Wiedervereinigung berücksichtigten. Diese wurde nicht adäquat durch Steuererhöhungen, sondern weitgehend auf Pump finanziert.

Was die Politik seit Langem an Begründungen für die jetzt geplante „Schuldenbremse" bietet, kann man nur noch als ökonomisches Nirwana bezeichnen. Da wird mit Polemik scheinargumentiert und Volksverdummung betrieben. Der Staatshaushalt mutiert dabei zu einem Privat-

\footnotetext{
Bremen, Berlin, Schleswig-Holstein, Sachsen-Anhalt und Saarland 
haushalt. Ein „privater Hausvater“ könne auch nicht permanent mehr ausgeben als er einnimmt. So wird - übrigens wie bei den Löhnen - einzelwirtschaftliche und gesamtwirtschaftliche Ökonomie (bewusst) verwechselt. Genauso wie viele Politiker offensichtlich nicht verstehen, dass Löhne, genauer Lohnstückkosten, nicht nur Arbeitskosten, sondern auch Arbeitseinkommen sind, verstehen sie nicht den gesamtwirtschaftlichen Zusammenhang einer Staatsverschuldung. Wo ein Schuldner ist, ist immer auch ein Gläubiger. Bei einer Staatsschuld wird, wie auch bei jeder privaten Schuld, nicht nur die Verbindlichkeit, die Schuld, vererbt, sondern ebenso die dahinterstehende Forderung, das Vermögen. Und dies inklusive der Zinsen. Die Zinszahlungen führen allerdings zu einer Umverteilung von unten nach oben, weil der Staat sich in der Regel bei vermögenden Staatsbürgern verschuldet, fällige Zinsen aber aus dem allgemeinen Steueraufkommen begleicht. Dennoch wird auch hier von Neoliberalen die Kausalität der Zinszahlungen auf den Kopf gestellt. Denn: Zinseinkommen entstehen dadurch, dass einzelne private Haushalte aufgrund ihrer hohen Einkommen in der Lage sind, Ersparnisse zu bilden. Aus der Staatsverschuldung folgt somit kein Gerechtigkeitsproblem, das nicht mit Blick auf die vorhandenen Einkommens- und Vermögensdisparitäten bereits bestanden hätte, also auch ohne Staatsverschuldung.

Ohne Schuldner könnte in einer Volkswirtschaft niemand Überschüsse erwirtschaften. Das Defizit des einen ist notwendigerweise der Überschuss des anderen. Am Anfang wirtschaftlicher Entwicklung steht immer der Kredit. Dies zeigen regelmäßig die Finanzierungssalden der Wirtschaftssektoren in der Volkswirtschaftli- chen Gesamtrechnung, die in der Summe immer gleich Null sind. Die privaten Haushalte bilden hier hohe Finanzierungsüberschüsse (Geldvermögensbildung minus Kreditaufnahme) - siehe diesbezüglich auch die hohe deutsche gesamtwirtschaftliche Sparquote bezogen auf das verfügbare Einkommen in Höhe von gut 11 \%. Insbesondere der Staat, neben den Unternehmen und dem Ausland, gleicht die Finanzierungsüberschüsse, die Forderungen als Kreditnehmer aus. Will ein Staat diese Überschüsse nicht, wie in der Vergangenheit, über Schulden zur Finanzierung öffentlicher Ausgaben abschöpfen und diese als staatliche Nachfrage in den Wirtschaftskreislauf zurückpumpen, so muss er, wie schon angeführt, mehr Steuern und Abgaben erheben und er muss vor allen Dingen die Massenarbeitslosigkeit beseitigen. Auf jeden Fall darf er die Steuern nicht senken. Schon gar nicht für Unternehmer und Vermögende. Und er darf auch nicht auf Vermögensteuern und z. B. auf eine Börsenumsatzsteuer verzichten und eine „Erbschaftsteuerreform" machen, die alles ist, nur keine Reform.

Auch das immer wieder von neoliberalen Politikern und Ökonomen betonte "Generationsargument" (wir würden mit Staatsverschuldung auf Kosten zukünftiger Generationen leben), ist ökonomisch lächerlich. Es ist vielmehr generationsübergreifend sinnvoll, die allokativ genutzte Schuldenaufnahme an das Ausmaß der öffentlichen Investitionen zu binden. Solange dann künftige Generationen Nutznießer der heute getätigten öffentlichen Investitionen sind, etwa zugunsten der Umwelt, der Bildung oder öffentlichen Infrastruktur, bietet nur die Staatsverschuldung die Möglichkeit, sie an der Finanzierung zu beteiligen. Hier gilt das richtige Prinzip „pay as you use". Der Finanzwissenschaftler Lorenz von Stein betonte schon 1878 zu Recht: „Ein Staat ohne Staatsschuld tut entweder zu wenig für seine Zukunft oder er fordert zu viel von seiner Gegenwart." Übrigens kommt das Scheinargument der Generationengerechtigkeit ausgerechtet von den Politikern und Parteien, die seit über dreiBig Jahren unfähig waren und es nach wie vor sind, sich mit den Problemen und Sorgen der Bürger und Bürgerinnen zu beschäftigen, die heute leben. Wie wäre es damit, die größten Gegenwartsprobleme anzugehen: die seit Langem bestehende „Geißel“ Massenarbeitslosigkeit, die Leid bringenden prekären Beschäftigungsverhältnisse sowie die in Deutschland bestehende Armut, inklusive der Kinderarmut? Diese wirklichen Probleme bekommt man allerdings nicht gelöst, wenn man, wie seit Mitte der 1970er Jahre, eine neoliberale Wirtschaftspolitik betreibt, die nur eins im Schilde führt und auch brutal umgesetzt hat: nämlich eine Umverteilung von unten nach oben.

Wer „Schuldenbremsen“ und den Abbau von Staatsverschuldung fordert, muss abschließend die Frage beantworten, wer denn dies künftig finanzieren soll: Das Kapital mit seinen Profitansprüchen oder die abhängig Beschäftigten, Rentner, Arbeitslose und/oder Sozialhilfeempfänger mit ihren Einkommensforderungen? Die Weiche zur Beantwortung dieser Frage ist in Deutschland mit der "Schuldenbremse“ gestellt worden. Die Antwort lautet: Mit Sicherheit nicht das Kapital! Man wird die Arbeitnehmer, Arbeitslosen und die Schwächsten der Gesellschaft noch mehr zur Kasse bitten und somit die soziale und ökonomische Spaltung in unserem Land vergrößern. 